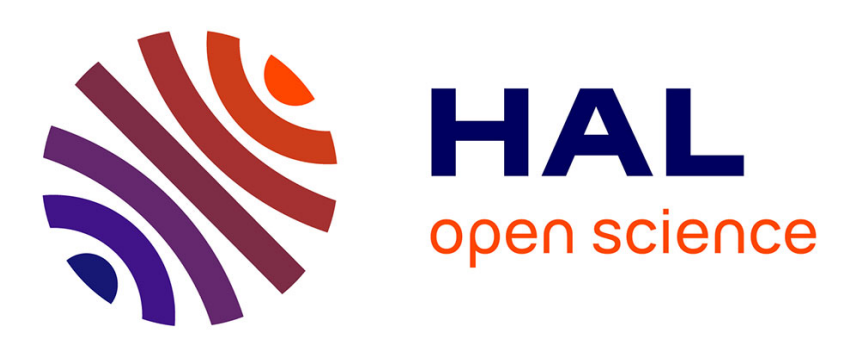

\title{
Genetics of Hepatocellular Carcinoma: Approaches to Explore Molecular Diversity
}

Stefano Caruso, Daniel O'brien, Sean Cleary, Lewis Roberts, Jessica Zucman-Rossi

\section{- To cite this version:}

Stefano Caruso, Daniel O'brien, Sean Cleary, Lewis Roberts, Jessica Zucman-Rossi. Genetics of Hepatocellular Carcinoma: Approaches to Explore Molecular Diversity. Hepatology, 2020, 10.1002/hep.31394 . hal-03097816

\section{HAL Id: hal-03097816 https: / hal.sorbonne-universite.fr/hal-03097816}

Submitted on 5 Jan 2021

HAL is a multi-disciplinary open access archive for the deposit and dissemination of scientific research documents, whether they are published or not. The documents may come from teaching and research institutions in France or abroad, or from public or private research centers.
L'archive ouverte pluridisciplinaire HAL, est destinée au dépôt et à la diffusion de documents scientifiques de niveau recherche, publiés ou non, émanant des établissements d'enseignement et de recherche français ou étrangers, des laboratoires publics ou privés. 


\section{Genetics of Hepatocellular Carcinoma: Approaches to Explore Molecular Diversity}

Authors: Stefano Caruso ${ }^{1}$, Daniel R. O’Brien², Sean P. Cleary ${ }^{3}$, Lewis R. Roberts ${ }^{4}$, Jessica Zucman-Rossi ${ }^{1,5}$.

Affiliations: ${ }^{1}$ Centre de Recherche des Cordeliers, INSERM, Sorbonne Université, Université de Paris, Université Paris 13, Functional Genomics of Solid Tumors laboratory, F-75006 Paris, France. ${ }^{2}$ Division of Biomedical Statistics and Informatics, Mayo Clinic College of Medicine and Science, Rochester, MN 55905, USA; ${ }^{3}$ Division of Hepatobiliary and Pancreatic Surgery, Mayo Clinic College of Medicine and Science, Rochester, MN 55905, USA; ${ }^{4}$ Division of Gastroenterology and Hepatology, Mayo Clinic College of Medicine and Science, Rochester, MN 55905, USA;

${ }^{5}$ European Hospital Georges Pompidou, AP-HP, F-75015, Paris, France

Potential conflict of interest: Dr. Roberts consults for, advises, and received grants from Exact Sciences. He consults for and received grants from Wako. He advises and received grants from Bayer and Gilead. He advises GRAIL, QED, and TAVEC and received grants from BTG, GlycoTest, RedHill, TARGET, and Ariad. Dr. Cleary consults for Ethicon and Olympus.

Funding: This publication was supported by Grant Numbers CA186566, CA 221205 and CA 210964 from the National Cancer Institute (NCI) and by INSERM, Ligue Nationale contre le Cancer (Equipe Labellisée), Labex OncoImmunology (investissement d'avenir), grant IREB, Coup d'Elan de la Fondation Bettencourt-Schueller, the SIRIC CARPEM, Raymond Rosen Award from the Fondation pour le Recherche Médicale, Prix René and Andrée Duquesne - Comité de Paris Ligue Contre le Cancer and Fondation Mérieux. S.C. was supported by a funding from Labex OncoImunology and CARPEM.

Correspondence: Lewis Roberts $\mathrm{MB} \mathrm{ChB}, \mathrm{PhD}$, Division of Gastroenterology and Hepatology Mayo Clinic College of Medicine and Science, 200 First Street SW, Rochester, Minnesota, 55905, USA. email: roberts.lewis@mayo.edu; and Jessica Zucman-Rossi, MD, PhD, Centre de Recherche des Cordeliers, INSERM, Sorbonne Université, Université de Paris, Université Paris 13, Functional Genomics of Solid Tumors laboratory, 15 rue de l'Ecole de Médecine, 75006 Paris, France. e-mail: jessica.zucman-rossi@inserm.fr.

\section{ORCID:}

Stefano Caruso: 0000-0002-6319-3642

Daniel R. O'Brien: 0000-0003-0740-7447

Sean P. Cleary: 0000-0002-5274-9983

Lewis R. Roberts: 0000-0001-7885-8574

Jessica Zucman-Rossi: 0000-0002-5687-0334 
Hepatocellular carcinomas (HCCs) are highly heterogeneous malignancies with different risk factors, including alcohol abuse, chronic hepatitis B virus (HBV) and hepatitis $\mathrm{C}$ virus (HCV) infection, fatty liver disease and other genetic disorders. Despite recent progress in HCC management, most are diagnosed at advanced stages, when therapeutic options are limited. Currently, multikinase inhibitors (sorafenib, lenvatinib, cabozantinib and regorafenib), human monoclonal antibodies (ramucirumab), and immune checkpoint inhibitors (nivolumab, pembrolizumab) are the only systemic therapies approved for the treatment of unresectable HCC (1). However, these drugs show low response rates and limited survival benefit of 2-3 months. Tumor heterogeneity, characterized by distinct molecular alterations in different sub-clones within each tumor or among tumors from different patients, contributes to drug resistance (2). Consequently, improved understanding of tumor heterogeneity and mechanisms of resistance to systemic treatments is required.

Advances in next-generation sequencing have increased our understanding of the molecular complexity of HCC, delineating a molecular landscape which includes recurrent genetic alterations that drive tumor expansion, as well as inherited variants that increase HCC risk. This review provides an overview of the genetic changes during HCC development and progression, with a special focus on the role of the tumor heterogeneity. We also discuss the latest advances in the establishment of preclinical models to investigate the molecular diversity of $\mathrm{HCC}$ and their use for developing effective personalized therapies.

\section{1- Genetic landscape in HCC \\ 1.1- Constitutional variations}

Accumulating evidence has demonstrated multifactorial susceptibility to HCC involving both genetic and environmental factors (3). Several constitutional single nucleotide polymorphisms (SNPs) have been associated with HCC risk, including predisposition to risk factors, severity of liver disease, malignant transformation, and tumor progression. Most variants were identified through case-control studies testing SNPs implicated in biological pathways affected during hepatocarcinogenesis, and include SNPs involved in iron metabolism (HFE1), inflammation (TNFA, IL1B, IL10, TGFB), oxidative stress (GSTM1, SOD2, MPO), cell cycle and DNA repair (MDM2, TP53, MTHFR, XRCC3). More recently, genome-wide association studies (GWAS) have examined associations between thousands of SNPs and risk of HCC. SNPs affecting different genes, including $D D X 18$ (encoding a putative RNA helicase), DEPDC5 (unknown function), MICA (immune regulation), GRIK1 (glutamate signaling), STAT4 (inflammation), and KIF1B (organelle and vesicular transport) have been associated with HCC (4). These studies were mainly conducted in Asian populations with chronic HBV or HCV infections using healthy individuals as controls, potentially resulting in selection bias (5). Few of these SNPs have been conclusively validated.

GWAS also identified SNPs in Patatin-like phospholipase domain-containing 3 (PNPLA3 rs738409) and Transmembrane 6 superfamily member 2 (TM6SF2 rs58542926) involved in lipid metabolism and fatty acid accumulation. Interestingly, these two SNPs were associated with Alcoholic Liver Disease (ALD) and Non-Alcoholic Fatty Liver Disease (NAFLD) and progression to cirrhosis (6,7). Both SNPs are also associated with HCC in patients with ALD or NAFLD (8), but their role in HCC development on HCV-related cirrhosis or other etiologies remains uncertain (9).

Interestingly, a loss-of-function variant of 17-beta-hydroxysteroid dehydrogenase 13 (HSD17B13 rs72613567) was initially associated with a reduced risk of NAFLD and ALD using GWAS(10) but subsequently reported as protective for HCC in patients with ALD (11) (Fig. 1A). These observations require further study.

None of the identified SNPs associated with risk of HCC show adequate odds ratios for introduction into clinical practice as predictive markers. However, future integration of genetic variants with clinical features may improve the existing risk-assessment models for HCC. 


\section{2- Somatic genomic alterations}

Accumulation of somatic genomic alterations in regulatory pathways is a major promoter of liver carcinogenesis. Whole-exome/genome sequencing have delineated the mutational landscape of HCC, identifying novel cancer driver genes and oncogenic pathways, reporting 50-70 non-silent mutations per tumor (12-14). The mutation rate remains stable across progressive stages, from early to advanced HCCs (15). Most mutations are in passenger genes, occurring randomly without functional consequences, while 2-6 mutations per tumor are driver mutations with a pivotal impact on tumor evolution. Aberrant telomerase reactivation through TERT promoter mutations, viral insertions into the TERT gene, TERT gene translocations or amplifications, is the most frequent somatic genomic alteration in $\mathrm{HCC}$ (16). Other genes frequently mutated in $\mathrm{HCC}$ are CTNNB 1, TP53, AXIN1, ARID1A, $A R I D 2, R B 1$, and NFE2L2. Many other genes are mutated at low frequency. Driver mutations mainly impact six crucial biological pathways, telomere maintenance, Wnt/B-catenin, cell cycle control, epigenetic regulation, oxidative stress, and the AKT/mTOR and MAP kinase pathways (Fig. 1B).

Differences in the mutation rates in driver genes seen in different studies may reflect differences in the etiologic risk factors in different populations. Accordingly, inactivating mutations of TP53 are enriched in HBV-related HCCs as well as HCCs in patients exposed to aflatoxin B1. Conversely, TERT promoter mutations and CTNNBI and ARIDIA inactivating mutations are related to HCC development in patients with ALD (12).

DNA copy number alterations (CNAs), including both gain and loss of DNA regions, are common genomic events in HCC. Recurrent focal amplifications commonly involve FGF19 and CCND1, both at the chromosome 11q13 locus. Interestingly, unlike CCND1, FGF19 amplification is not sufficient to cause over-expression. Thus, the sole presence of FGF19 amplification cannot predict sensitivity to drugs acting on the FGF19/FGFR4 signaling pathway (17). Other frequent focal amplifications in HCC affect TERT, VEGFA, MYC, and MET. Recurrent homozygous deletions involve AXIN1, CDKN2A, PTEN, RB1, and RPS6KA3 $(12,18)$. Co-occurrence between TERT promoter and CTNNB1 alterations and also between $A X I N 1$ and RPS6KA3 mutations has been reported. It has also been shown that CTNNB1 and TP53 or CTNNB1 and AXIN1 alterations are mutually exclusive in HCC $(12,14,15)$.

\section{3- Viral integration}

Accumulation of somatic mutations induced by longstanding inflammation is the pro-carcinogenic mechanism for most pre-disposing conditions for HCC, including ALD, NAFLD, and HCV, in which cancer develops in the setting of cirrhosis. In HBV-associated HCC, tumors can develop in the absence of cirrhosis, indicating alternative oncogenic mechanisms. Integration of HBV, a doublestranded (ds) DNA virus, into the host genome mediates carcinogenesis through stable expression of pro-oncogenic viral proteins as well as direct effects of viral integration.

Expression of integrated viral genes including mutated HBV surface antigen proteins is associated with increased stress response in the endoplasmic reticulum and stimulation of clonal expansion (19). Overexpression of the HBx protein has numerous pro-oncogenic effects including p53 inactivation, inhibition of apoptosis, stimulation of cell proliferation and induction of stem-cell like properties.

Integration of $\mathrm{HBV}$ into the host genome can result in somatic alterations that predispose the host to carcinogenesis. HBV integration into the host genome can induce a wide spectrum of genetic alterations including single nucleotide variants, small insertions, small deletions, translocations, copy number variants, altered expression of host proteins, the creation of chimeric fusion transcripts, and generalized genomic instability (20). All of these genetic alterations are associated with increased cancer risk. The primary mechanism of HBV integration is thought to be aberrant repair of random dsDNA break repair events. Analyses of integration sites have also implicated micro-homology mediated repair pathways (21). Sequence analysis of infected non-tumor liver tissue supports both mechanisms, revealing a relatively random pattern of viral integrations predominantly within intronic regions, as well as a subset of recurrent integrations, specifically in the FN1 gene (22). In contrast, integrations in HCCs favor coding and promoter regions of host genes. Furthermore, integrations 
appear more frequent in regions of open chromatin, repetitive sequences (such as $\mathrm{CpG}$ islands and Alu elements), known fragile sites and telomeres. Analysis of fusion mRNA transcripts demonstrate a preferential breakpoint between $1500-2000 \mathrm{bp}$ of the viral genome within close proximity of the direct repeat 1 (DR1) sequence at the 3' end of the HBx gene where the core gene and viral enhancer are also located (23). Recurrent integrations in HBV associated HCC have been detected in many genes including TERT, MLL4, CCNE1, SENP5 and ROCK1 (24) (Fig. 1C). Such integrations are often associated with marked increases in expression of the target gene, presumably due to integration of viral enhancer or de-repression of regulatory promoter sequences. Integrations can also create oncogenic viral-host gene fusions. A chimeric protein of HBx-LINE1 resulted in Wnt activation (25). Other chimeric fusion targets described include ESPL1 (26), CCNA2 and SERCA1 (27).

Recently, integrated adeno-associated virus 2 (AAV2) sequences have also been demonstrated in HCC (28). AAV is a single-stranded DNA virus that requires co-infection with another virus, most commonly human herpes virus (HHV) types 4 and 6 , for infection. AAV is endemic, with $30-80 \%$ of the population demonstrating seropositivity. AAV DNA positivity in HCC is higher in females and persons without underlying cirrhosis. Recurrent AAV2 integrations targeting TERT, CCNA2, CCNE1, GLI1/INHBE, TNFSF 10 and KMT2B have been identified near the transcriptional start site or in the 5' upstream region, resulting in overexpression of full-length or mutated oncogenic proteins (29) (Fig. 1). AAV and HBV integrations are both strongly associated with chromosomal instability characterized by copy number variants within $1 \mathrm{Mb}$ of integration sites (24). The mechanisms by which this wide spectrum of genomics alterations drives carcinogenesis in AAV and HBV associated $\mathrm{HCC}$ are under active investigation.

\section{2- Heterogeneity in $\mathrm{HCC}$}

\section{1- Intra-tumoral heterogeneity}

Advances in high-throughput sequencing technologies have provided insight into the complex architecture of HCC. There is substantial intra-tumoral heterogeneity, with subpopulations of cancer cells exhibiting distinct molecular and biological characteristics within the same tumor. Intra-tumoral heterogeneity has been demonstrated at morphological, histological (30), DNA ploidy (31), protein, genomic and epigenomic levels. The clonal evolution model proposed by Nowell in 1976 posits that tumor evolution is a dynamic process following malignant transformation and expansion of the founding clone, in which accumulation of sequential molecular alterations under selective pressure leads to formation of a heterogeneous tumor mass (32) (Fig. 2A). The resulting spatial molecular diversity within the tumor is characterized by trunk (or clonal) alterations ubiquitously present in all cancer cells and private, branch or sub-clonal alterations occurring in distinct tumor regions (33). The main driver mutations in HCC, affecting TERT, CTNNB1 and TP53, occur in early carcinogenesis and are clonal and shared by all malignant tumor cells $(30,34)$. Clonal trunk mutations have also been shown in druggable genes such as $K I T, S Y K$, and $P I K 3 C$ A, providing new therapeutic opportunities in persons carrying these alterations (35). Conversely, passenger mutations that do not directly affect tumor development occur throughout tumor progression and are mainly sub-clonal.

Besides the traditional Darwinian model of clonal selection during tumor progression, other mechanisms for intra-tumor heterogeneity have been proposed. In the "Big Bang" model of tumor evolution, it is proposed that after malignant transformation, a single expansion of different heterogeneous sub-clones occurs, with the majority of alterations occurring at an early stage when the tumor is relatively small (36) (Fig. 2B). Some studies in HCC have been consistent with a nonDarwinian model of intra-tumoral evolution (37).

A third model of intra-tumoral heterogeneity implicates cancer stem cells (CSCs) (38). These are pluripotent self-renewing cells responsible for tumor initiation and growth maintenance. In this model of hierarchically organized tumors, CSCs differentiate into multiple cell populations within the tumor. Tumor cellular plasticity can shift from a differentiated to an undifferentiated state. Accordingly, 
following specific genetic events a differentiated cell can dedifferentiate and re-acquire CSC features, thus generating a new hierarchical clone which further increases intra-tumoral diversity (39) (Fig. 2C).

The tumor microenvironment (TME) also plays key roles in promoting tumor progression and intratumoral heterogeneity. The TME comprises immune cells, fibroblasts, and endothelial cells that modulate the process of tumorigenesis, contributing to tumor diversity. Thus, epigenetic aberrations in the underlying liver cirrhosis/fibrosis could establish a "field effect" causing predisposition to HCC (40). Further, single-cell RNA sequencing has shown that release of pro-angiogenic factors such as vascular endothelial growth factor A (VEGFA) in tumors with high intra-tumoral heterogeneity induces both TME polarization and T lymphocyte dysfunction, promoting tumor progression (41). The positive results of the phase III study combining the immune checkpoint inhibitor atezolizumab with the anti-VEGFA humanized monoclonal antibody bevacizumab validate the importance of targeting the TME in patients with unresectable HCC (NCT03434379).

\section{2- Inter-tumoral heterogeneity}

Another layer of complexity of cell diversity in HCC relies in the inter-tumoral heterogeneity distinguishing different hepatic lesions. Multifocal HCC may result from both metachronous multicentric occurrence (MO) of a de novo clone or intrahepatic metastatic (IM) spread. Although these two types of lesions are different in clinical features and prognostic outcomes, distinguishing between multi-centric and metastatic tumors remains challenging. Conventionally, it is presumed that recurrences of the primary tumor occur early, within the first 2 years after resection, whereas recurrences arising from de novo liver carcinogenesis on cirrhosis occur late, 2 years or more after resection (42). Molecular approaches to discriminate the origin of multiple lesions include studies of chromosomal aberrations, LOH, comparative genomic hybridization, methylation specific PCR and hepatitis B virus integration patterns (43). Recently, new "omics" approaches have improved our understanding of inter-tumoral heterogeneity, including genomics, transcriptomics, proteomics, and metabolomics $(44,45)$. Phylogenetic comparison of primary tumors to IM resected 1 year after the index surgery found that IM show higher molecular intra-tumoral heterogeneity than their primary tumors, indicating that metastatic tumors progress faster; however, the divergence between the IM and primary tumor was relatively small in comparison with the differences in sectors of the primary tumor. This suggests a minimal need for further adaptation of IM to the microenvironment. The increased heterogeneity of IM may be a consequence of rapid growth fueled by the enhanced capacity for liver regeneration, which promotes rapid diversification and enhanced genetic variability of the IM (34). By contrast, MO share very few mutations with primary tumors. Thus, MOs originating and developing independently from primary tumors should be sampled and evaluated separately (46). Common epigenetic aberrations and other mutagenic factors could contribute to a "field effect" that promotes liver tumorigenesis and the occurrence of multiple lesions (40,46). In addition, shared mutations in protein-coding genes or structural variant breakpoints assessed by whole genome sequencing can discriminate IM from MO (42).

The presence of tumor heterogeneity, either within the same tumor mass or between different lesions, is one of the main causes of treatment resistance (47). A better evaluation of tumor heterogeneity through the use of multiregional sampling is therefore necessary to identify the optimal therapeutic strategy. Indeed, the existence of targetable mutations assessed by individual biopsies could be limited to only one tumor region and consequently ineffective as a biomarker of treatment response. For example, the low success rate of sorafenib may be explained by the presence of sorafenib-targeted alterations only in some tumor lesions and not clonally throughout an HCC (44).

The heterogeneity of the local immune microenvironment is less pronounced than that of the tumor mass, thus targeting the immune TME may represent a relevant therapeutic solution for multifocal HCC (45). Nevertheless, recent immunogenomic approaches on multiregional HCC samples have shown a close relationship between regional adaptive immune response and tumor heterogeneity (48). 


\section{3- Interpatient tumor heterogeneity}

A third dimension of tumor heterogeneity refers to the genetic and molecular variability existing between tumors from different patients, also known as interpatient heterogeneity. Studies defining a consensus molecular classification of HCCs by integrating molecular data with etiological, clinical and pathological features classify HCC into two major molecular classes $(14,49,50)$.

The first "proliferation class" is characterized by poorly-differentiated tumors with a more aggressive phenotype and vascular invasion, a higher likelihood of HBV etiology, and higher alpha-fetoprotein levels. Genetically, this class is enriched in TP53 mutations, FGF19/CCND1 amplifications, chromosomal instability, global DNA hypomethylation and activation of cell cycle/proliferation signaling pathways including AKT/mTOR, MET, and RAS/MAP kinase $(4,49)$. Within the proliferation class, two other subtypes are identified: a "progenitor" subclass related to the G1 transcriptomic group characterized by expression of hepatic progenitor markers; and a more aggressive subclass associated with the G2-G3 groups and activation of the TGF-beta pathway $(49,50)$.

The "nonproliferation class" is defined by less aggressive more differentiated tumors with normal hepatocyte-like features. These tumors are associated with HCV- or alcohol-related HCC and chromosomal stability. This class can also be divided into two further subtypes: a subclass characterized by the activation of the Wnt/B-catenin pathway, enriched in CTNNB1 mutations and associated to the G5-G6 transcriptomic groups; and a more heterogeneous subclass related to the G4 group with inflammation-related genomic traits $(49,51)$.

Thus, HCCs at the same clinical stage can have significantly different molecular, clinical and prognostic features (15). The Barcelona Clinic Liver Cancer (BCLC) staging system is currently used to predict prognosis and guide treatment decisions. Although several molecular signatures to predict HCC prognosis have been proposed, none has been implemented into clinical practice (52-55). In addition, most available studies have used samples from surgical resections, consequently genomic data from advanced tumors are limited. It has been shown that similar to earlier stage HCCs, biopsies from advanced HCCs exhibit molecular diversity, with enrichment in the G3 transcriptomic group, a poor prognostic score and higher frequency of TP53, RBI and SF3B1 mutations (15). Further studies are needed of larger cohorts to better understand interpatient heterogeneity and the potential therapeutic consequences.

\section{3- New preclinical models to reconstruct molecular diversity in $\mathrm{HCC}$ in relation to treatment response}

Restricted access to HCC samples, especially for more advanced stages, is one of the main limitations to understanding molecular heterogeneity and identifying new effective therapeutic strategies. Accordingly, the use of preclinical experimental models able to capture the genetic diversity of human HCC and identify potential biomarkers of treatment response is critical. Here, we describe commonly used preclinical HCC models including tumor-derived cell lines, patient-derived tumor xenografts (PDTXs) and patient-derived tumor organoids (PDTOs), and their roles in studies predicting treatment response (Fig. 3).

\section{1- Cell models}

Human tumor-derived cell lines have been among the most broadly used experimental models for studying tumor biology and drug-biomarker discovery. Pharmacogenomic studies using large pancancer panels of cell lines, including CCLE (Cancer Cell Line Encyclopedia), CTRP (Cancer Therapeutics Response Portal), and GDSC (Genomics of Drug Sensitivity in Cancer), showed the ability of in vitro models to identify molecular alterations revealing druggable vulnerabilities (5659). These studies confirmed that molecularly characterized cell lines can recapitulate the diversity of human tumors, facilitating translation of discoveries into clinical practice. Unfortunately, HCC cell lines were poorly represented in these datasets. 
Unsupervised classification of 20 human HCC cell lines using microarrays identified 2 subgroups analogous to the "HC" subgroup with a hepatocyte-like phenotype and "HB" subgroup with a progenitor-like phenotype, respectively. Only HB subtype cell lines were sensitive to the Src/Abl inhibitor dasatinib (60). Transcriptomic profiles of 25 liver cancer cell lines were also comparable to S1 and S2 human HCC subtypes (61).

Integrated multi-omics analysis including whole-exome, RNA, microRNA sequencing and quantification of 126 proteins with pharmacological sensitivity profiles of 31 anti-cancer compounds in 34 liver cancer cell lines showed the most common molecular alterations of HCC cell lines were similar to those of more proliferative and aggressive HCC primary tumors, corresponding to the "proliferation class" (17). Correspondingly, gene expression profiles identified three robust subgroups of liver cancer cell lines, CL1, CL2, CL3 closely associated with the Boyault G1-G3 and Hoshida S1-S2 HCC subclasses (17) (Table 1)(https://lccl.zucmanlab.com).

Notably, the "nonproliferation class" of HCC tumors is not represented among currently available liver cancer cell lines. To overcome this obstacle, a Liver Cancer Model Repository (LIMORE) including 50 new patient-derived cell models established from 49 Chinese HCCs and 31 publicly available HCC cell lines has been established to investigate tumor heterogeneity and identify novel gene-drug associations (62). All Hoshida HCC subclasses (S1-S3) are represented in the LIMORE models.

Patient-derived primary cancer cells derived from spatially distinct samples of different tumor regions can be also used to model intra-tumoral heterogeneity and identify the best therapeutic approach to drug resistance (63).

Despite the major limitations of in vitro systems, including the absence of a TME and 3D tissue architecture, human tumor-derived cell lines remain valuable preclinical models of the molecular heterogeneity of $\mathrm{HCC}$ and can identify predictive biomarkers of drug response that may guide clinical decision making. Importantly, genomic and transcriptomic profiles of HCC cell lines show substantial stability during in vitro culture with the accumulation of passages over time (64). In the future, the acquisition of an increasing number of cell lines using new culture techniques should further improve the capability of these models to reconstruct the molecular heterogeneity of HCC.

\section{2- Mouse models}

Because of their physiological and molecular similarities to humans, mice provide some of the best experimental models for tumor growth and progression in vivo. However, modeling both the underlying chronic liver disease and $\mathrm{HCC}$ in mice remains challenging. Approaches used to model HCC in mice include genetically engineered mice (GEM), carcinogenic chemicals and implantation models (65). GEM models induce HCC formation through techniques that activate oncogenes or inactivate tumor suppressor genes. Chemically-induced HCC models use carcinogens that induce liver injury, irreversible structural DNA alterations, and expansion of preneoplastic clones. Diethylnitrosamine (DEN), the most widely used liver carcinogen, acts by alkylating DNA, promoting oxidative stress and inducing genomic alterations. Although particular mutations are frequently observed in chemical models, such as mutation of the $R A S$ oncogene, reproducible induction of specific genetic alterations remains difficult, limiting their use in modeling tumor heterogeneity. Comparative genomic analyses of mouse GEM or chemical models versus human HCC have shown that some models are similar to low grade human HCCs with fewer oncogenic alterations $(53,66)$. Implantation models are heterotopic, injected subcutaneously, or orthotopic, injected directly into the liver or indirectly into the spleen. Xenograft models using human-derived cell lines or tumors prevent bias due to species-specific mutations, providing more relevant models for assessing drug response in vivo. To limit murine immune reactions against human tumors, HCC cells/tumors are commonly implanted into immunocompromised mice such as athymic nude mice or non-obese-diabetic-severe combined immunodeficient (NOD/SCID) mice. Xenograft models established from HCC cell lines are accessible and easy to manipulate. These models allow evaluation of the in vivo effects of long-term treatment and non-invasive monitoring of tumor growth using 
luciferase expressing cell lines (65). Patient derived tumor xenografts (PDTX) can be established from resected cancers or needle biopsies. A large pharmacogenomic study of approximatively 1000 PDTX from different cancer types confirmed substantial ability to predict drug response and resistance (67). For HCC, the overall PDTX engraftment rate is relatively low, approximatively $20 \%$. Studies of HCC PDTX have shown similar histology, gene expression, and drug responses to the matched original tumors (68). HCC biopsy-derived PDTXs also maintained the clinicohistopathological and molecular features of the HCC biopsies over generations of retransplantation. To provide a comprehensive resource facilitating the use of PDTX models, a publicly available database containing 116 PDTX models from Chinese HCC patients, designated PDXliver, was recently assembled, including molecular, clinical, and pharmacological profiles and providing an excellent tool for investigating tumor heterogeneity and drug response in vivo.

Due to their lack of a complete immune system, PDTX models established using immunocompromised mice are not suitable for studying the TME or the effects of immunotherapy. Humanized mouse models transplanted with human immune cells can address this deficiency and accurately simulate the human TME. Humanized HCC PDTX models with a type I human leucocyte antigen matched human immune system have been shown to have an immune system influenced by the tumors, with an exhausted phenotype characterized by decreases in leucocytes, reduced production of human pro-inflammatory cytokines and increased expression of exhaustion markers. Unlike other PDTX models, these humanized PDTXs were sensitive to the immune checkpoint inhibitors pembrolizumab and ipilimumab (69).

\section{3- Organoid models}

Although both 2D in vitro culture and PDTXs have improved our understanding of HCC complexity, there are still major limitations in the use of these models to investigate tumor heterogeneity and to test drug efficacy. While monolayer-cultured cancer cells fail to mimic the 3D tumor architecture and cell-cell or cell-matrix interactions, PDTX models display a low engraftment rate and require long periods to be established, limiting their use in high-throughput screening. Advances in in vitro techniques include new 3D cultures, termed organoids, which mimic the in vivo tissue architecture and functions. Organoids are established using stem/progenitor cells isolated from adult tissues or pluripotent stem cells (PSCs), including embryonic stem cells (ESCs) or induced pluripotent stem cells (iPSCs) (70). The first murine and human liver-derived organoids used progenitor cells derived from adult bile duct bipotent cells and an optimized culture medium $(71,72)$. Subsequently, long-term $3 \mathrm{D}$ culture systems from both murine and human primary hepatocytes or iPSCs were proposed (7375). These protocols were adapted to establish primary liver tumor organoids in vitro, also called tumoroids or patient-derived tumor organoids (PDTO). Long term in vitro expansion of PDTOs from resected $\mathrm{HCC}$, cholangiocarcinoma $(\mathrm{CC})$, and mixed $\mathrm{HCC} / \mathrm{CC}$ accurately recapitulated the histological and molecular features of the respective patients over time, with more than $90 \%$ of genomic alterations of primary tumors retained in the corresponding PDTOs cultured for less than 2 months and more than $80 \%$ retained after 4 months (76).

Similar to PDTXs, long term HCC PDTO cultures can be established from needle biopsies with a success rate over $20 \%$. Despite the low success rate compared toother cancers, the establishment of PDTO HCC models from biopsies could provide more relevant preclinical models for advanced unresectable tumors. HCC PDTOs are also able to generate PDTX maintaining the morphology and the molecular diversity of the corresponding tumors (77).

The molecular similarity between tumor organoids and matching primary tumors makes PDTOs excellent models for drug screening and biomarker discovery, including studies modeling intra- and inter-tumoral heterogeneity $(76,78)$.

Whether PDTO models are able to fully represent tumor heterogeneity remains to be clarified. The low efficiency rate of $20-40 \%$ suggests that only tumors containing more cancer progenitor or stem cells can establish organoids. Moreover, whether PDTO can recapitulate the TME, including immune cells and blood vessels, is still unclear. Initial evidence suggests that co-cultures from both epithelial 
and non-epithelial tumor tissue, including stromal and immune cells, could recreate a more accurate tumor architecture (79).

\section{Conclusion and future perspective}

Tumor heterogeneity of HCC has major implications for optimizing drug therapy. Although advances in genomics have improved our understanding of the complexity of HCC, much remains to be explored. Notably, most studies exploring HCC heterogeneity have analyzed surgical specimens, not the more advanced tumors requiring systemic therapy. Further, sampling tumors with a single biopsy may limit identification of targetable sub-clonal alterations present in particular tumor regions. Therefore, studies of larger HCC cohorts and single cell approaches to investigating tumor heterogeneity and drug response are needed. Accordingly, emerging rapid autopsy protocols as well as the analysis of circulating tumor DNA in the context of liquid biopsy are promising alternative non-invasive procedures to capture the extent of molecular diversity and identify new effective therapeutic strategies that address tumor heterogeneity.

Thus far, studies have primarily focused on how genetic diversity affects drug sensitivity in small series of HCCs, and little is known about the effects of treatment on molecular heterogeneity. Preclinical models able to capture the heterogeneity of HCC tumors will be crucial tools for elucidating these effects. PDTXs and PDTOs represent optimal models to investigate tumor heterogeneity and identify biomarkers predictive of treatment response. However, the low efficiency of model establishment and lack of an authentic TME, particularly the immune context, limit the translation of preclinical findings to clinical studies. The development of organoid co-culture systems or humanized PDTX models may overcome these limitations, facilitating the identification of novel therapies for HCC.

\section{References}

1. Villanueva A. Hepatocellular Carcinoma. N. Engl. J. Med. 2019;380:1450-1462.

2. Alizadeh AA, Aranda V, Bardelli A, Blanpain C, Bock C, Borowski C, et al. Toward understanding and exploiting tumor heterogeneity. Nat. Med. 2015;21:846-853.

3. Caruso S, Calderaro J, Letouzé E, Nault J-C, Couchy G, Boulai A, et al. Germline and somatic DICER1 mutations in familial and sporadic liver tumors. J. Hepatol. 2017;66:734-742.

4. Zucman-Rossi J, Villanueva A, Nault J-C, Llovet JM. Genetic Landscape and Biomarkers of Hepatocellular Carcinoma. Gastroenterology. 2015;149:1226-1239.e4.

5. Nahon P, Zucman-Rossi J. Single nucleotide polymorphisms and risk of hepatocellular carcinoma in cirrhosis. J. Hepatol. 2012;57:663-674.

6. Romeo S, Kozlitina J, Xing C, Pertsemlidis A, Cox D, Pennacchio LA, et al. Genetic variation in PNPLA3 confers susceptibility to nonalcoholic fatty liver disease. Nat. Genet. 2008;40:1461-1465.

7. Kozlitina J, Smagris E, Stender S, Nordestgaard BG, Zhou HH, Tybjærg-Hansen A, et al. Exomewide association study identifies a TM6SF2 variant that confers susceptibility to nonalcoholic fatty liver disease. Nat. Genet. 2014;46:352-356.

8. Buch S, Stickel F, Trépo E, Way M, Herrmann A, Nischalke HD, et al. A genome-wide association study confirms PNPLA3 and identifies TM6SF2 and MBOAT7 as risk loci for alcohol-related cirrhosis. Nat. Genet. 2015;47:1443-1448.

9. Yang J, Trépo E, Nahon P, Cao Q, Moreno C, Letouzé E, et al. PNPLA3 and TM6SF2 variants as risk factors of hepatocellular carcinoma across various etiologies and severity of underlying liver diseases. Int. J. Cancer. 2019;144:533-544.

10. Abul-Husn NS, Cheng X, Li AH, Xin Y, Schurmann C, Stevis P, et al. A Protein-Truncating HSD17B13 Variant and Protection from Chronic Liver Disease. N. Engl. J. Med. 2018;378:10961106.

11. Yang J, Trépo E, Nahon P, Cao Q, Moreno C, Letouzé E, et al. A 17-Beta-Hydroxysteroid Dehydrogenase 13 Variant Protects From Hepatocellular Carcinoma Development in Alcoholic Liver Disease. Hepatol. Baltim. Md. 2019;70:231-240. 
12. Schulze K, Imbeaud S, Letouzé E, Alexandrov LB, Calderaro J, Rebouissou S, et al. Exome sequencing of hepatocellular carcinomas identifies new mutational signatures and potential therapeutic targets. Nat. Genet. 2015;47:505-511.

13. Ahn S-M, Jang SJ, Shim JH, Kim D, Hong S-M, Sung CO, et al. Genomic portrait of resectable hepatocellular carcinomas: implications of RB1 and FGF19 aberrations for patient stratification. Hepatol. Baltim. Md. 2014;60:1972-1982.

14. Cancer Genome Atlas Research Network. Electronic address: wheeler@bcm.edu, Cancer Genome Atlas Research Network. Comprehensive and Integrative Genomic Characterization of Hepatocellular Carcinoma. Cell. 2017;169:1327-1341.e23.

15. Nault J-C, Martin Y, Caruso S, Hirsch TZ, Bayard Q, Calderaro J, et al. Clinical Impact of Genomic Diversity From Early to Advanced Hepatocellular Carcinoma. Hepatol. Baltim. Md. 2019; 16. Nault J-C, Ningarhari M, Rebouissou S, Zucman-Rossi J. The role of telomeres and telomerase in cirrhosis and liver cancer. Nat. Rev. Gastroenterol. Hepatol. 2019;16:544-558.

17. Caruso S, Calatayud A-L, Pilet J, La Bella T, Rekik S, Imbeaud S, et al. Analysis of Liver Cancer Cell Lines Identifies Agents With Likely Efficacy Against Hepatocellular Carcinoma and Markers of Response. Gastroenterology. 2019;157:760-776.

18. Guichard C, Amaddeo G, Imbeaud S, Ladeiro Y, Pelletier L, Maad IB, et al. Integrated analysis of somatic mutations and focal copy-number changes identifies key genes and pathways in hepatocellular carcinoma. Nat. Genet. 2012;44:694-698.

19. Wang H-C, Wu H-C, Chen C-F, Fausto N, Lei H-Y, Su I-J. Different types of ground glass hepatocytes in chronic hepatitis B virus infection contain specific pre-S mutants that may induce endoplasmic reticulum stress. Am. J. Pathol. 2003;163:2441-2449.

20. Bonilla Guerrero R, Roberts LR. The role of hepatitis B virus integrations in the pathogenesis of human hepatocellular carcinoma. J. Hepatol. 2005;42:760-777.

21. Verdin H, D'haene B, Beysen D, Novikova Y, Menten B, Sante T, et al. Microhomologymediated mechanisms underlie non-recurrent disease-causing microdeletions of the FOXL2 gene or its regulatory domain. PLoS Genet. 2013;9:e1003358.

22. Furuta M, Tanaka H, Shiraishi Y, Unida T, Imamura M, Fujimoto A, et al. Characterization of HBV integration patterns and timing in liver cancer and $\mathrm{HBV}$-infected livers. Oncotarget. 2018;9:25075-25088.

23. Jiang Z, Jhunjhunwala S, Liu J, Haverty PM, Kennemer MI, Guan Y, et al. The effects of hepatitis $B$ virus integration into the genomes of hepatocellular carcinoma patients. Genome Res. 2012;22:593-601.

24. Ding D, Lou X, Hua D, Yu W, Li L, Wang J, et al. Recurrent targeted genes of hepatitis B virus in the liver cancer genomes identified by a next-generation sequencing-based approach. PLoS Genet. 2012;8:e1003065.

25. Lau C-C, Sun T, Ching AKK, He M, Li J-W, Wong AM, et al. Viral-human chimeric transcript predisposes risk to liver cancer development and progression. Cancer Cell. 2014;25:335-349.

26. Hu B, Huang W, Wang R, Zang W, Su M, Li H, et al. High Rate of Detection of Human ESPL1HBV S Fusion Gene in Patients With HBV-related Liver Cancer: A Chinese Case-Control Study. Anticancer Res. 2020;40:245-252.

27. Chiu Y-T, Wong JKL, Choi S-W, Sze KMF, Ho DWH, Chan L-K, et al. Novel pre-mRNA splicing of intronically integrated HBV generates oncogenic chimera in hepatocellular carcinoma. J. Hepatol. 2016;64:1256-1264.

28. Nault J-C, Datta S, Imbeaud S, Franconi A, Mallet M, Couchy G, et al. Recurrent AAV2-related insertional mutagenesis in human hepatocellular carcinomas. Nat. Genet. 2015;47:1187-1193.

29. La Bella T, Imbeaud S, Peneau C, Mami I, Datta S, Bayard Q, et al. Adeno-associated virus in the liver: natural history and consequences in tumour development. Gut. 2019;

30. Friemel J, Rechsteiner M, Frick L, Böhm F, Struckmann K, Egger M, et al. Intratumor heterogeneity in hepatocellular carcinoma. Clin. Cancer Res. Off. J. Am. Assoc. Cancer Res. 2015;21:1951-1961. 
31. Bou-Nader M, Caruso S, Donne R, Celton-Morizur S, Calderaro J, Gentric G, et al. Polyploidy spectrum: a new marker in HCC classification. Gut. 2019;

32. Nowell PC. The clonal evolution of tumor cell populations. Science. 1976;194:23-28.

33. Dhanasekaran R, Nault J-C, Roberts LR, Zucman-Rossi J. Genomic Medicine and Implications for Hepatocellular Carcinoma Prevention and Therapy. Gastroenterology. 2019;156:492-509.

34. Zhai W, Lim TK-H, Zhang T, Phang S-T, Tiang Z, Guan P, et al. The spatial organization of intra-tumour heterogeneity and evolutionary trajectories of metastases in hepatocellular carcinoma. Nat. Commun. 2017;8:4565.

35. Lin D-C, Mayakonda A, Dinh HQ, Huang P, Lin L, Liu X, et al. Genomic and Epigenomic Heterogeneity of Hepatocellular Carcinoma. Cancer Res. 2017;77:2255-2265.

36. Sottoriva A, Kang H, Ma Z, Graham TA, Salomon MP, Zhao J, et al. A Big Bang model of human colorectal tumor growth. Nat. Genet. 2015;47:209-216.

37. Ling S, Hu Z, Yang Z, Yang F, Li Y, Lin P, et al. Extremely high genetic diversity in a single tumor points to prevalence of non-Darwinian cell evolution. Proc. Natl. Acad. Sci. U. S. A. 2015; 112:E6496-6505.

38. Prasetyanti PR, Medema JP. Intra-tumor heterogeneity from a cancer stem cell perspective. Mol. Cancer. 2017;16:41.

39. Zheng H, Pomyen Y, Hernandez MO, Li C, Livak F, Tang W, et al. Single-cell analysis reveals cancer stem cell heterogeneity in hepatocellular carcinoma. Hepatol. Baltim. Md. 2018;68:127-140. 40. Ding X, He M, Chan AWH, Song QX, Sze SC, Chen H, et al. Genomic and Epigenomic Features of Primary and Recurrent Hepatocellular Carcinomas. Gastroenterology. 2019;

41. Ma L, Hernandez MO, Zhao Y, Mehta M, Tran B, Kelly M, et al. Tumor Cell Biodiversity Drives Microenvironmental Reprogramming in Liver Cancer. Cancer Cell. 2019;36:418-430.e6.

42. Furuta M, Ueno M, Fujimoto A, Hayami S, Yasukawa S, Kojima F, et al. Whole genome sequencing discriminates hepatocellular carcinoma with intrahepatic metastasis from multi-centric tumors. J. Hepatol. 2017;66:363-373.

43. Xie D-Y, Fan H-K, Ren Z-G, Fan J, Gao Q. Identifying Clonal Origin of Multifocal Hepatocellular Carcinoma and Its Clinical Implications. Clin. Transl. Gastroenterol. 2019;10:e00006. 44. Xu LX, He MH, Dai ZH, Yu J, Wang JG, Li XC, et al. Genomic and transcriptional heterogeneity of multifocal hepatocellular carcinoma. Ann. Oncol. Off. J. Eur. Soc. Med. Oncol. 2019;

45. Zhang Q, Lou Y, Yang J, Wang J, Feng J, Zhao Y, et al. Integrated multiomic analysis reveals comprehensive tumour heterogeneity and novel immunophenotypic classification in hepatocellular carcinomas. Gut. 2019;68:2019-2031.

46. Xue R, Li R, Guo H, Guo L, Su Z, Ni X, et al. Variable Intra-Tumor Genomic Heterogeneity of Multiple Lesions in Patients With Hepatocellular Carcinoma. Gastroenterology. 2016;150:998-1008. 47. Dagogo-Jack I, Shaw AT. Tumour heterogeneity and resistance to cancer therapies. Nat. Rev. Clin. Oncol. 2018;15:81-94.

48. Losic B, Craig AJ, Villacorta-Martin C, Martins-Filho SN, Akers N, Chen X, et al. Intratumoral heterogeneity and clonal evolution in liver cancer. Nat. Commun. 2020;11:291.

49. Boyault S, Rickman DS, de Reyniès A, Balabaud C, Rebouissou S, Jeannot E, et al. Transcriptome classification of HCC is related to gene alterations and to new therapeutic targets. Hepatol. Baltim. Md. 2007;45:42-52.

50. Hoshida Y, Nijman SMB, Kobayashi M, Chan JA, Brunet J-P, Chiang DY, et al. Integrative transcriptome analysis reveals common molecular subclasses of human hepatocellular carcinoma. Cancer Res. 2009;69:7385-7392.

51. Calderaro J, Ziol M, Paradis V, Zucman-Rossi J. Molecular and histological correlations in liver cancer. J. Hepatol. 2019;71:616-630.

52. Hoshida Y, Moeini A, Alsinet C, Kojima K, Villanueva A. Gene signatures in the management of hepatocellular carcinoma. Semin. Oncol. 2012;39:473-485.

53. Lee J-S, Heo J, Libbrecht L, Chu I-S, Kaposi-Novak P, Calvisi DF, et al. A novel prognostic subtype of human hepatocellular carcinoma derived from hepatic progenitor cells. Nat. Med. 
2006;12:410-416.

54. Villanueva A, Hoshida Y, Battiston C, Tovar V, Sia D, Alsinet C, et al. Combining clinical, pathology, and gene expression data to predict recurrence of hepatocellular carcinoma. Gastroenterology. 2011;140:1501-1512.e2.

55. Nault J-C, De Reyniès A, Villanueva A, Calderaro J, Rebouissou S, Couchy G, et al. A hepatocellular carcinoma 5-gene score associated with survival of patients after liver resection. Gastroenterology. 2013;145:176-187.

56. Barretina J, Caponigro G, Stransky N, Venkatesan K, Margolin AA, Kim S, et al. The Cancer Cell Line Encyclopedia enables predictive modelling of anticancer drug sensitivity. Nature. 2012;483:603-607.

57. Basu A, Bodycombe NE, Cheah JH, Price EV, Liu K, Schaefer GI, et al. An interactive resource to identify cancer genetic and lineage dependencies targeted by small molecules. Cell. 2013;154:1151-1161.

58. Iorio F, Knijnenburg TA, Vis DJ, Bignell GR, Menden MP, Schubert M, et al. A Landscape of Pharmacogenomic Interactions in Cancer. Cell. 2016;166:740-754.

59. Behan FM, Iorio F, Picco G, Gonçalves E, Beaver CM, Migliardi G, et al. Prioritization of cancer therapeutic targets using CRISPR-Cas9 screens. Nature. 2019;568:511-516.

60. Finn RS, Aleshin A, Dering J, Yang P, Ginther C, Desai A, et al. Molecular subtype and response to dasatinib, an Src/Abl small molecule kinase inhibitor, in hepatocellular carcinoma cell lines in vitro. Hepatol. Baltim. Md. 2013;57:1838-1846.

61. Hirschfield H, Bian CB, Higashi T, Nakagawa S, Zeleke TZ, Nair VD, et al. In vitro modeling of hepatocellular carcinoma molecular subtypes for anti-cancer drug assessment. Exp. Mol. Med. 2018;50:e419.

62. Qiu Z, Li H, Zhang Z, Zhu Z, He S, Wang X, et al. A Pharmacogenomic Landscape in Human Liver Cancers. Cancer Cell. 2019;36:179-193.e11.

63. Gao Q, Wang Z-C, Duan M, Lin Y-H, Zhou X-Y, Worthley DL, et al. Cell Culture System for Analysis of Genetic Heterogeneity Within Hepatocellular Carcinomas and Response to Pharmacologic Agents. Gastroenterology. 2017;152:232-242.e4.

64. Qiu Z, Zou K, Zhuang L, Qin J, Li H, Li C, et al. Hepatocellular carcinoma cell lines retain the genomic and transcriptomic landscapes of primary human cancers. Sci. Rep. 2016;6:27411.

65. Brown ZJ, Heinrich B, Greten TF. Mouse models of hepatocellular carcinoma: an overview and highlights for immunotherapy research. Nat. Rev. Gastroenterol. Hepatol. 2018;15:536-554.

66. Dow M, Pyke RM, Tsui BY, Alexandrov LB, Nakagawa H, Taniguchi K, et al. Integrative genomic analysis of mouse and human hepatocellular carcinoma. Proc. Natl. Acad. Sci. U. S. A. 2018;115:E9879-E9888.

67. Gao H, Korn JM, Ferretti S, Monahan JE, Wang Y, Singh M, et al. High-throughput screening using patient-derived tumor xenografts to predict clinical trial drug response. Nat. Med. 2015;21:1318-1325.

68. Hu B, Li H, Guo W, Sun Y-F, Zhang X, Tang W-G, et al. Establishment of a hepatocellular carcinoma patient-derived xenograft platform and its application in biomarker identification. Int. J. Cancer. 2019;

69. Zhao Y, Shuen TWH, Toh TB, Chan XY, Liu M, Tan SY, et al. Development of a new patientderived xenograft humanised mouse model to study human-specific tumour microenvironment and immunotherapy. Gut. 2018;67:1845-1854.

70. Prior N, Inacio P, Huch M. Liver organoids: from basic research to therapeutic applications. Gut. 2019;68:2228-2237.

71. Huch M, Dorrell C, Boj SF, van Es JH, Li VSW, van de Wetering M, et al. In vitro expansion of single Lgr5+ liver stem cells induced by Wnt-driven regeneration. Nature. 2013;494:247-250.

72. Huch M, Gehart H, van Boxtel R, Hamer K, Blokzij1 F, Verstegen MMA, et al. Long-term culture of genome-stable bipotent stem cells from adult human liver. Cell. 2015;160:299-312.

73. Hu H, Gehart H, Artegiani B, LÖpez-Iglesias C, Dekkers F, Basak O, et al. Long-Term Expansion 
of Functional Mouse and Human Hepatocytes as 3D Organoids. Cell. 2018;175:1591-1606.e19. 74. Peng WC, Logan CY, Fish M, Anbarchian T, Aguisanda F, Álvarez-Varela A, et al. Inflammatory Cytokine TNF $\alpha$ Promotes the Long-Term Expansion of Primary Hepatocytes in 3D Culture. Cell. 2018; 175:1607-1619.e15.

75. Takebe T, Sekine K, Enomura M, Koike H, Kimura M, Ogaeri T, et al. Vascularized and functional human liver from an iPSC-derived organ bud transplant. Nature. 2013;499:481-484.

76. Broutier L, Mastrogiovanni G, Verstegen MM, Francies HE, Gavarró LM, Bradshaw CR, et al. Human primary liver cancer-derived organoid cultures for disease modeling and drug screening. Nat. Med. 2017;23:1424-1435.

77. Nuciforo S, Fofana I, Matter MS, Blumer T, Calabrese D, Boldanova T, et al. Organoid Models of Human Liver Cancers Derived from Tumor Needle Biopsies. Cell Rep. 2018;24:1363-1376.

78. Li L, Knutsdottir H, Hui K, Weiss MJ, He J, Philosophe B, et al. Human primary liver cancer organoids reveal intratumor and interpatient drug response heterogeneity. JCI Insight. 2019;4.

79. Dijkstra KK, Cattaneo CM, Weeber F, Chalabi M, van de Haar J, Fanchi LF, et al. Generation of Tumor-Reactive T Cells by Co-culture of Peripheral Blood Lymphocytes and Tumor Organoids. Cell. 2018;174:1586-1598.e12. 
Table 1. Transcriptomic classification of the liver cancer cell lines among different studies.

\begin{tabular}{|c|c|c|c|c|c|c|c|c|}
\hline Cell line & Sex & Histology & HBV & HCV & Ethnicity & \begin{tabular}{|c} 
Finn et al., \\
Hepatology \\
2013
\end{tabular} & $\begin{array}{l}\text { Hirschfield et } \\
\text { al., Exp Mol } \\
\text { Med } 2018 \\
\end{array}$ & $\begin{array}{c}\text { Caruso et al., } \\
\text { Gastroenterology } \\
2019\end{array}$ \\
\hline & & & & & & Array & Array & RNA sequencing \\
\hline Hep3B & Male & $\mathrm{HCC}$ & Yes & No & Black & $\mathrm{HC}$ & S2 & CL1 \\
\hline HepG2 & Male & $\mathrm{HB}$ & No & No & Caucasian & $\mathrm{HC}$ & S2 & CL1 \\
\hline HLE & Male & $\mathrm{HCC}$ & No & No & Asian & HB & S1 & CL3 \\
\hline HLF & Male & $\mathrm{HCC}$ & No & No & Asian & $\mathrm{HB}$ & S1 & CL3 \\
\hline Huh1 & Male & $\mathrm{HCC}$ & Yes & na & Asian & $\mathrm{HC}$ & S2 & CL1 \\
\hline Huh7 & Male & $\mathrm{HCC}$ & No & No & Asian & $\mathrm{HC}$ & S2 & CL1 \\
\hline $\mathrm{JHH} 2$ & Male & $\mathrm{HCC}$ & No & $n a$ & Asian & $\mathrm{HB}$ & S1 & CL2 \\
\hline $\mathrm{JHH} 4$ & Male & $\mathrm{HCC}$ & No & Yes & Asian & $\mathrm{HB}$ & S2 & CL3 \\
\hline JHH5 & Male & $\mathrm{HCC}$ & No & $n a$ & Asian & $\mathrm{HC}$ & S2 & CL1 \\
\hline JHH6 & Female & $\mathrm{HCC}$ & No & Yes & Asian & HB & S1 & CL3 \\
\hline $\mathrm{JHH} 7$ & Male & $\mathrm{HCC}$ & Yes & $n a$ & Asian & $\mathrm{HC}$ & S2 & CL1 \\
\hline $\mathrm{JHH} 1$ & Male & $\mathrm{HCC}$ & No & Yes & Asian & $\mathrm{HC}$ & S1 & Unclassified \\
\hline Li7 & Male & $\mathrm{HCC}$ & No & No & Asian & $n a$ & S2 & CL2 \\
\hline PLC/PRF5 & Male & $\mathrm{HCC}$ & Yes & No & African & $\mathrm{HC}$ & S2 & CL1 \\
\hline SKHEP1 & Male & $\mathrm{HCC}$ & No & na & Caucasian & HB & S1 & $n a$ \\
\hline SNU182 & Male & $\mathrm{HCC}$ & Yes & No & Asian & HB & S1 & CL3 \\
\hline SNU387 & Female & $\mathrm{HCC}$ & Yes & No & Asian & HB & S1 & CL3 \\
\hline SNU398 & Male & $\mathrm{HCC}$ & Yes & No & Asian & $\mathrm{HC}$ & S2 & CL3 \\
\hline SNU423 & Male & $\mathrm{HCC}$ & Yes & No & Asian & $H B$ & S1 & CL3 \\
\hline SNU449 & Male & $\mathrm{HCC}$ & Yes & No & Asian & $H B$ & S1 & CL3 \\
\hline SNU475 & Male & $\mathrm{HCC}$ & Yes & No & Asian & HB & S1 & CL3 \\
\hline SNU761 & Male & $\mathrm{HCC}$ & Yes & $n a$ & Asian & $n a$ & S1 & CL2 \\
\hline SNU878 & Female & $\mathrm{HCC}$ & Yes & $n a$ & Asian & $n a$ & S1 & CL2 \\
\hline SNU886 & Male & $\mathrm{HCC}$ & Yes & $n a$ & Asian & $n a$ & S1 & $\mathrm{CL} 2$ \\
\hline
\end{tabular}

Note: Only cell lines present in at least two studies were reported.

Abbreviation: $n a$, not available. 


\section{A Constitutional variations}

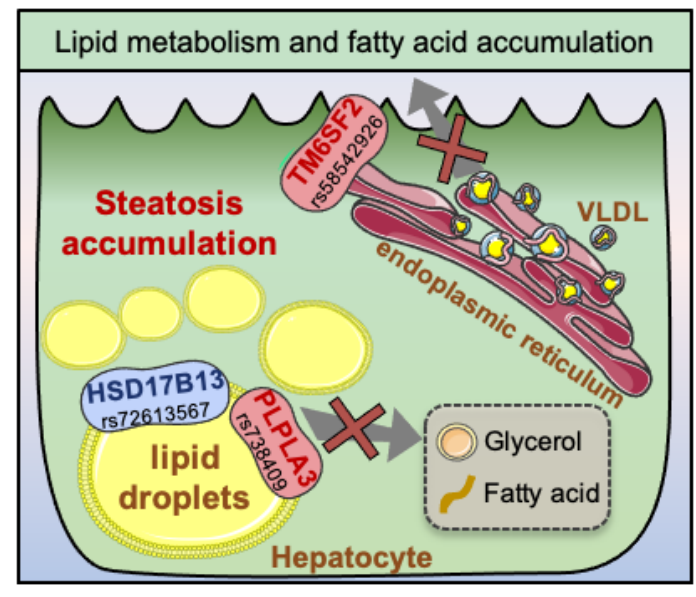

\section{Viral integration}

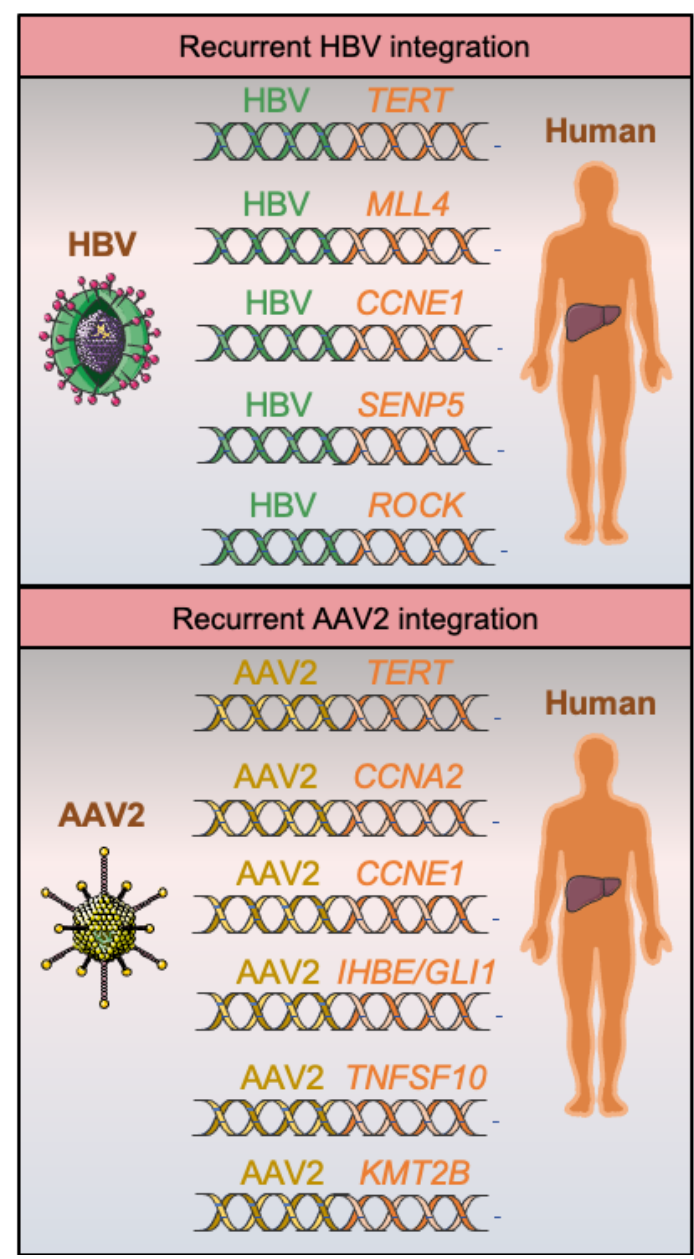

B

Somatic alterations

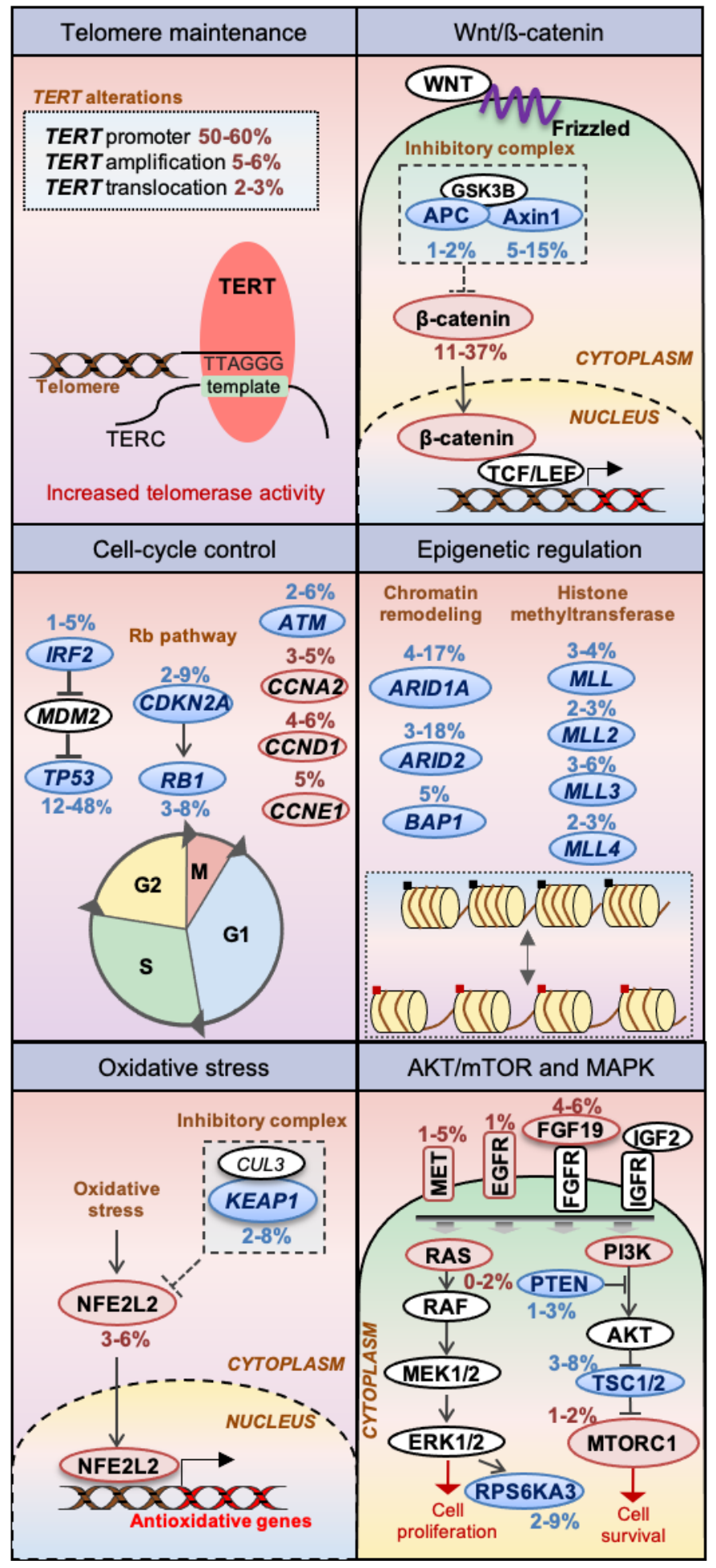

Figure 1. Graphical representation of the main genetic events in HCC. We represented the major inherited variants (A) and somatic alterations in signaling pathways (B) involved in liver carcinogenesis and the recurrent integrations in HBV or AAV2 associated HCC (C). Activating mutations of oncogenes/SNPs promoting HCC development and inactivating mutations in tumor suppressors/SNPs protecting from HCC are represented in red and blue, respectively. 
A

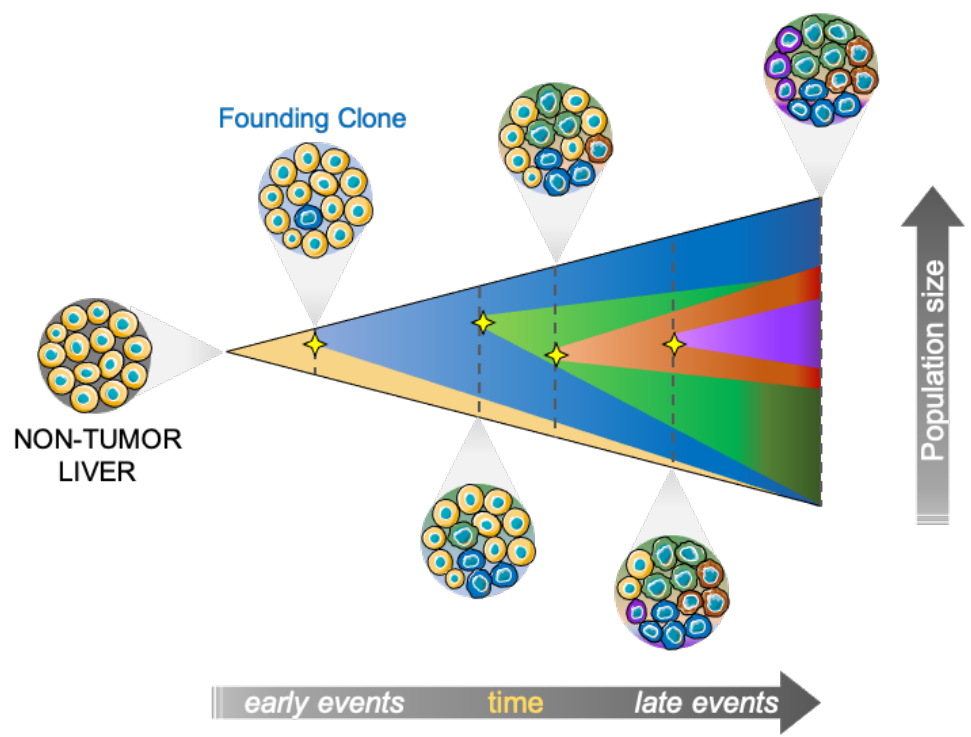

B

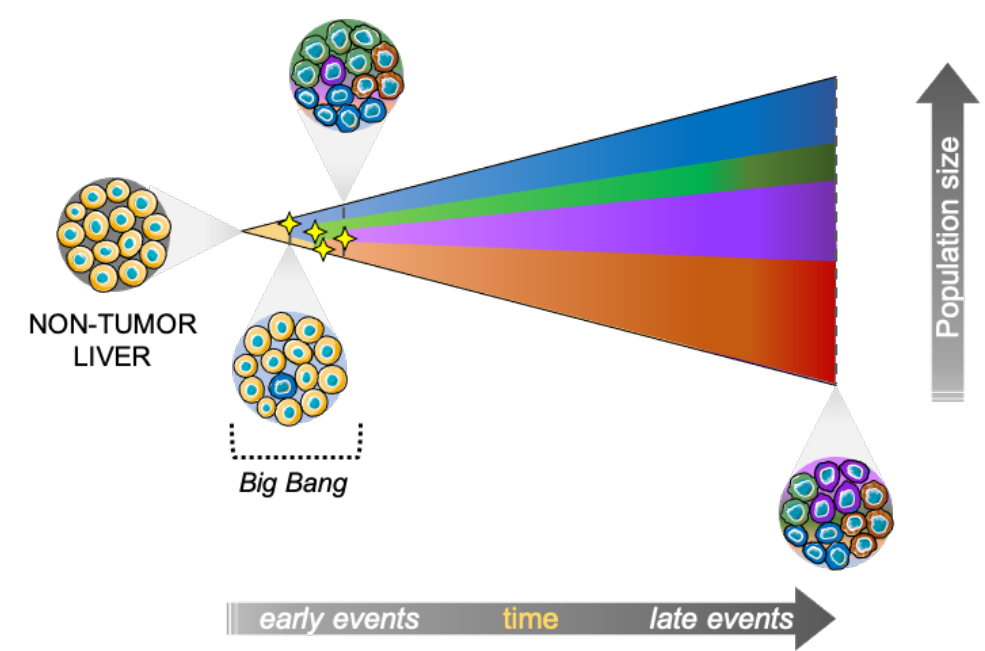

C
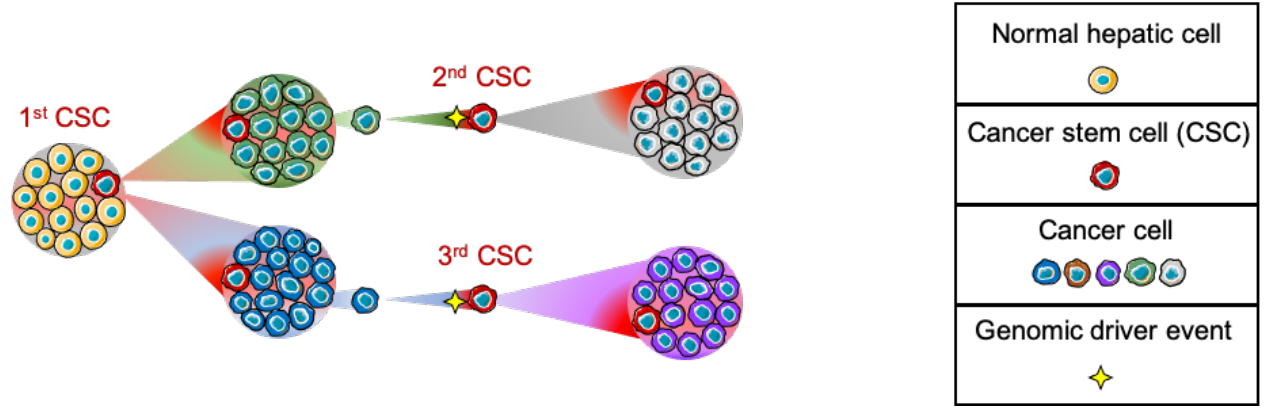

Figure 2. Schematic illustration of different models of intra-tumoral heterogeneity. (A) Clonal evolution model with the accumulation of sequential molecular alterations leading to formation of a heterogeneous tumor mass. (B) non-Darwinian model of intra-tumoral evolution with the majority of alterations occurring at an early stage when the tumor is relatively small. (C) In the cancer stem cells model the CSCs differentiate into multiple cell populations within the tumor but following specific genetic events a differentiated cancer cell can also re-acquire CSC features and further increases intra-tumoral diversity. 


\section{Drug Screening}
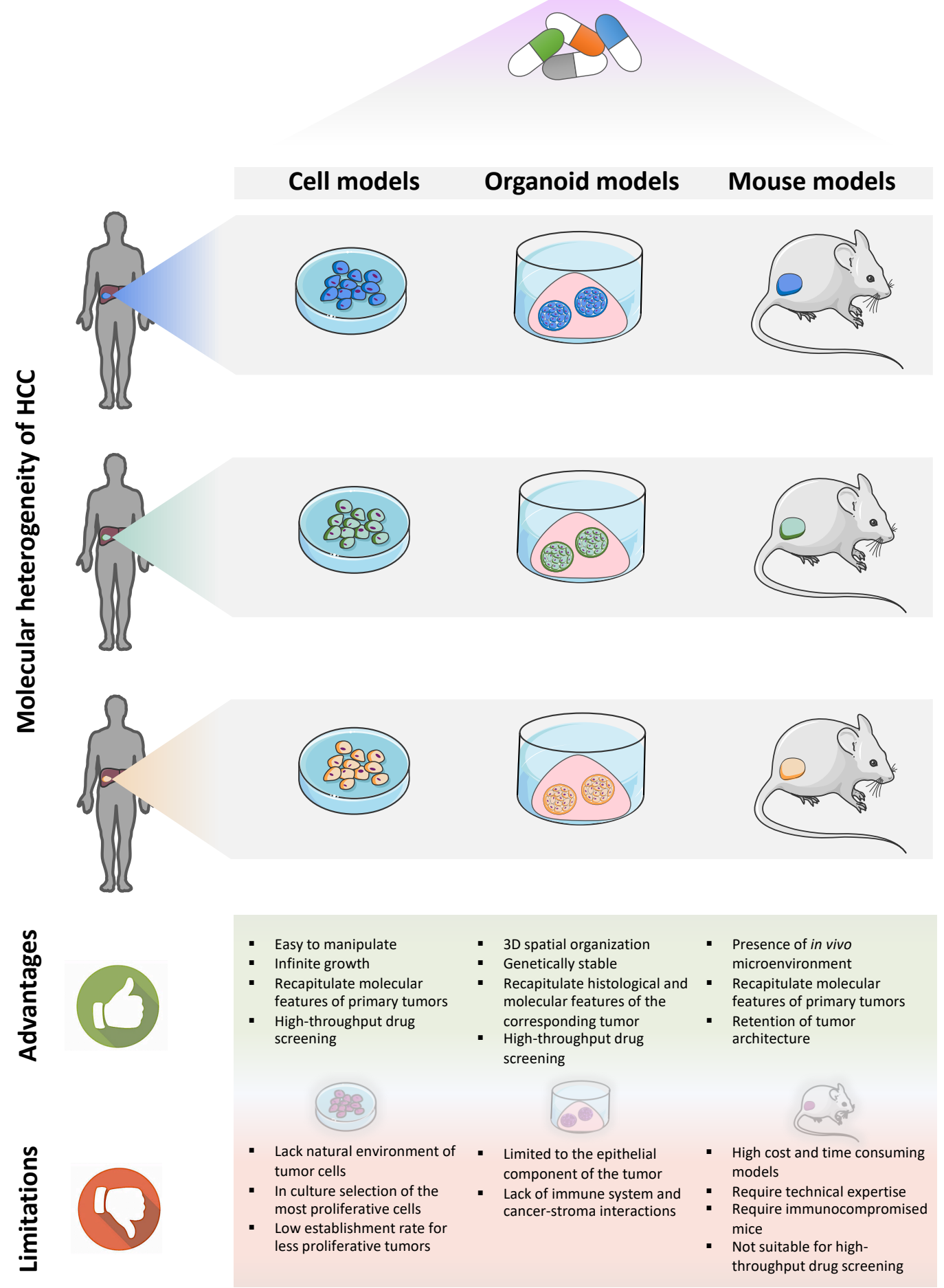

Figure 3. Preclinical models to study the molecular diversity in HCC. Representation of the 3 preclinical models currently available for the study of the molecular diversity of HCC, including cell, organoid and mouse models. Advantages and the current limitations for each of these models are reported at the bottom of the figure. 